\title{
An Atypical Etiology of Anosmia: Case Report and Literature Review
}

\author{
*Diogo Pereira Nunes, Eduard Janz and Andreas Naumann \\ Department of Otolaryngology - Head and Neck Surgery, Klinikum Bremen-Mitte, Germany
}

Submission: December 18, 2016; Published: January 06, 2017

*Corresponding author: Diogo Pereira Nunes, Department of Otolaryngology - Head and Neck Surgery, Klinikum Bremen-Mitte, St.-Jürgen-Str. 1, 28177 Bremen, Germany, Email: pereiranunes.diogo@gmail.com

\begin{abstract}
Sinonasal undifferentiated carcinoma (SNUC) is a rare tumor that typically presents with nonspecific symptoms such as epistaxis, nasal obstruction, headache and facial pain. The neoplasm typically arises in the ethmoid and maxillary sinus and up to $50 \%$ of patients present orbital, skull base or brain involvement. Invasive growth can lead to manifestations such as proptosis, cranial nerve palsies and visual disturbances. We report an atypical presentation of this tumor. Due to the nonspecificity of the manifestationsand to the fact that SNUC is highly aggressive, up to $70-90 \%$ of patients present with stage T4 disease and overall mortality is high. Therapeutic approaches vary between studies and none has demonstrated a coherent advantage. The purpose of this paper is to alert about an atypical presentation of this tumor and to reinforce the importance of a complete and correct patient evaluation in order to avoid future misdiagnosis. The obvious consequence is an advanced local disease at presentation with a poor prognosis. The nonspecificity of the symptoms increases the probability for it to be ignored for a long time.
\end{abstract}

Keywords: SNUC; Sinonasal undifferentiated carcinoma; Nasal tumors; Nasal malignant tumors

\section{Introduction and Case Report}

A 52-year-old Caucasian female with complaints of chronic nasal obstruction and anosmia was conservative treated for 2 years. As past medical history, the patient reported the surgical removal of a melanoma of the right breast. She was sent to our tertiary care center after developing a forehead swelling and decreased visual acuity. Contrast-enhanced CT-Scan and MRI (Figures 1 \& 2) showed a large nasal cavity tumor, achieving both frontal sinuses, with bone expansion and erosion, and infiltrating the anterior cranial fossa. A nasal endoscopic guided biopsy (right ethmoidal and frontal sinuses) was performed under general anesthesia and the histologic findings revealed a sinonasal undifferentiated carcinoma (SNUC), EBV negative. According to the current TNM classification of sinonasal tumors, the neoplasm was classified as cT4b.

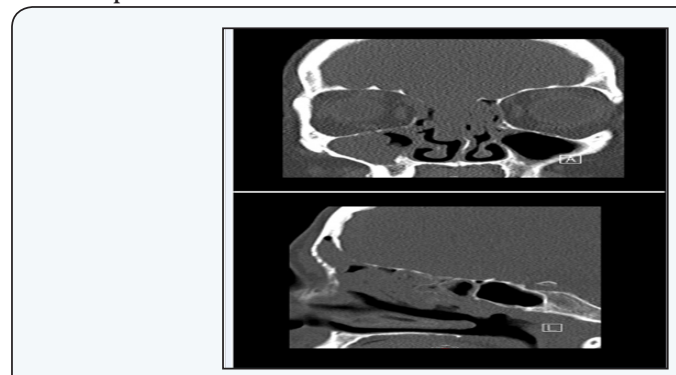

Figure 1: Contrast-enhanced CT-Scan showing a large nasal cavity tumor.

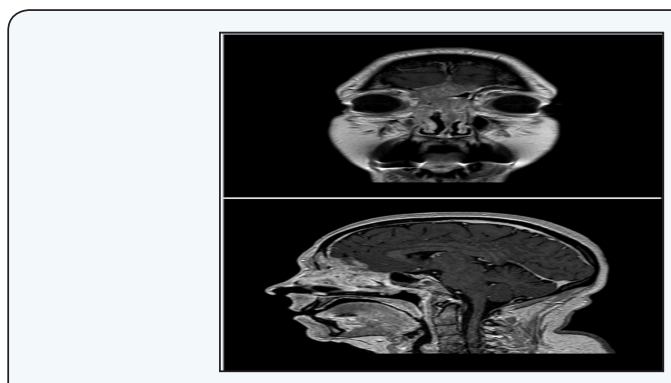

Figure 2: SNUC infiltrating the anterior cranial fossa (contrastenhanced MRI, T1).

Further staging excluded cervical or distant metastases. This tumor was also classified as C, according to the Kadish System. The case was presented on a multidisciplinary meeting and, because an $\mathrm{R} 0$ resection would not be possible, a decision was made against a surgical treatment. A primary radiochemotherapy was recommended and initiated. The medication of choice for the chemotherapy was cisplatin. This manuscript intends to warn about anuncommon etiology of anosmia and, following the presented case, to revise the literature concerning anosmia and sinonasal undifferentiated carcinoma.

\section{Discussion}

The term anosmia is used to describe the complete absence of useful smelling ability [1]. Olfactory disorders have four 


\section{Global Journal of Otolaryngology}

major causes: upper respiratory infections, sinonasal diseases, head trauma and neurodegenerative illnesses (Parkinson's disease, Alzheimer's disease, etc.). Upper respiratory infections (URI), especially viral, and sinonasal diseases (allergic rhinitis, chronic rhinosinusitis with or without nasal polyps, sinonasal benign or malignant tumors) together are responsible for 32 to $72 \%$ of olfactory disorders, depending on the chosen studies. Sinonasal diseases alone are responsible for $14 \%$ of olfactory disorders [2,3]. Smell loss due to URI has no available therapy and, on the other hand, some sinonasal disorders often respond considerably to proper treatment. In spite of the importance of this distinction, this is not always easy, due to the nonspecific findings presented by some patients.

As a result, the etiology is often incorrectly thought to be postviral or idiopathic. Scott et al. found a significant number of patients with sinonasal diseases inadequately diagnosed with post-URI olfactory loss. It is then imperative to collect a correct patient's history and to perform an accurate clinical examination $[2,3]$. According to Seiden, a systemic steroid administration is useful distinguishing between a conductive loss (sinonasal disease) that will improve and a sensorineural loss (URI) that will not respond $[1,2,4]$. The physical examination should include a complete head and neck examination, with a special attention given to nasal endoscopy [5]. Seiden et al. [2,3,5] showed that anterior rhinoscopy alone was only able to diagnose sinonasal disease as a cause of smell loss in $51 \%$ of patients with the disease, while nasal endoscopy missed the diagnosis in $9 \%$. Isolated septal deviation and moderate turbinate hypertrophy are of limited predictive value finding a etiology for smell loss [3].

If the patient's history, clinical examination and olfactory tests suggest obstructive sinonasal disease (nasal polyps, benign or malignant tumors) or severe chronic rhinosinusitis, radiological imaging is then indicated. CT-Scan is the gold standard for the identification of sinonasal disorders and it is indispensable for a correct surgical planning [1,5]. Scott et al. [2] showed that a normal examination does not exclude nasal or sinus disease as a cause of olfactory loss (reports of $24 \%$ of positive CT-Scan findings in patients with normal examinations). MRI is useful to add important information when malignant tumors are suspected (nasal, paranasal sinuses, skull base or intracranial). The most common nasal tumors are inverting papillomas, adenomas, squamous cell carcinomas and esthesioneuroblastomas $[1,3]$.

Malignant sinonasal tumors are rare. These correspond to less than $1 \%$ of all neoplasms and only $3 \%$ of all head and neck tumors. The majority are squamous carcinomas and their variants (55\%), followed by nonepithelial neoplasms $(20 \%)$, glandular tumors (15\%), undifferentiated carcinomas (7\%) and miscellaneous tumors (3\%) [6]. The sinonasal undifferentiated carcinoma (SNUC) was first described by Frierson et al. [7] in 1986. Between its description and 2009, 167 cases were reported. It is a rare tumor characterized by a poor differentiation and to distinguish it from other tumors of the sinonasal tract can be challenging [8]. Although some cases have occurred after radiotherapy for nasopharyngeal carcinomas, the etiology remains uncertain and the neoplasm is usually negative for Epstein-Barr virus [6,9].

For the definitive diagnosis, microscopy and immunohistochemical criteria are used [8]. The histologic appearance is characterized by a hypercellular proliferation with varied growth [9]. The majority of patients with SNUC presents with epistaxis, nasal obstruction, headache and facial pain. Due to the nonspecificity of the symptoms and to the fact that SNUC is a highly aggressive tumor, up to $70-90 \%$ of patients present with stage T4 disease and overall mortality is high. The neoplasm typically arises in the ethmoid and maxillary sinus and up to $50 \%$ of patient's present orbital, skull base or brain involvement. Invasive growth can lead to manifestations such as proptosis, cranial nerve palsies and visual disturbances. In spite of the frequent locally advanced disease at presentation, at this time only $5-13 \%$ of patients have nodal metastasis [8-13].

Earlier reports presented consistently very poor outcomes. However, current series show an overall survival between 40 and 75\% [11-14]. Neck metastases in advanced local disease are a poor prognostic sign [8]. In what concerns the treatment, the infrequency of this tumor, the challenge in establishing a diagnosis and the heterogeneity in therapy approaches contribute to a lack of consensus [6]. Therapeutic approaches vary between studies and none has demonstrated a coherent advantage. When the neoplasm is limited to the nasal cavity, surgery and adjuvant radiotherapy as well as definitive radiotherapy can be the treatment of choice. The preferred therapy for advanced local disease remains however unclear, debated and extremely variable depending on the chosen report [6-8]. Regardless the initial treatment of choice, it seems to be unanimous that a multimodality therapy (between surgery, radiotherapy and chemotherapy) improves survival outcomes.

According to Reiersen et al. $[6,8,12,15]$, the patients treated with a single modality had increased recurrence and mortality rates when compared with patients with a similar tumor stage. However, when comparing single modalities, surgery alone (when possible) appears to have a survival advantage over other options. Current information available does not demonstrate a clear advantage of preoperative over postoperative adjuvant therapy. Other options such as proton beam radiation therapy need further investigation. The current literature, despite not presenting directly the percentage of anosmia cases caused by sinonasal tumors, shows that $14 \%$ of olfactory disorders have sinonasal diseases as etiology. Evidently, only a part of all olfactory disorders refers to anosmia and only a part of all sinonasal diseases refers to sinonasal tumors (benign or malignant). Malignant sinonasal tumors are rare and correspond to less than $1 \%$ of all neoplasms and $3 \%$ of all head and neck tumors. Undifferentiated carcinomas are only $7 \%$ of all malignant sinonasal tumors. These percentages show how improbable it is 
to have a SNUC as etiology for anosmia. However, and due to the frequent late presentation of patients with this tumor and the consequent high mortality rate, it is crucial to keep this entity in mind. A complete head and neck examination, including nasal endoscopy is of an extreme importance, to avoid a misdiagnosis.

\section{Conclusion}

The term anosmia is used to describe the complete absence of useful smelling ability [1]. Sinonasal undifferentiated carcinoma is a rare tumor that typically presents with nonspecific symptoms such as epistaxis, nasal obstruction, headache and facial pain. This case demonstrated that anosmia might also be a symptom. The nonspecificity of the symptoms increases the probability for it to be ignored for a long time. The purpose of this paper is to alert about an atypical presentation of this neoplasm and to reinforce the importance of a complete nasal cavity evaluation, eventually accompanied by radiological imaging, in order to avoid future misdiagnosis. The obvious consequence is an advanced local disease at presentation with a poor prognosis.

\section{Acknowledgement}

Author contributions: Drs Nunes, Janz and Naumann had full access to all the data in the study and take responsibility for the integrity of the data and the accuracy of the data analysis.

\section{Study concept and design: Diogo Nunes.}

Acquisition of data: Diogo Nunes; Eduard Janz.

Analysis and interpretation of data: Diogo Nunes.

Drafting of the manuscript: Diogo Nunes.

Critical revision of the manuscript for important intellectual content: All authors.

Administrative, technical, or material support: All authors.

Study supervision: Andreas Naumann.

Conflict of interest disclosures: None reported.

Sources of funding and support: None reported.

Previous Presentation: No previous presentation.

\section{References}

1. Allis TJ, Leopold DA (2012) Smell and taste disorders. Facial Plast Surg Clin N Am 20(1):93-111.

2. Seiden AM, Duncan HJ (2001) The diagnosis of a conductive olfactory loss. The Laryngoscope 111(1): 9-14.

3. Malaty J, Malaty IAC (2013) Smell and taste disorders in primary care. Am Fam Physician 88(12): 852-859.

4. Martinez-Devesa P, Patiar S (2011) Oral steroids for nasal polyps. Cochrane Database Syst Rev (7): CD005232.

5. Holbrook EH, Leopold DA (2003) Anosmia: diagnosis and management. Curr Opin Otolaryngol Head Neck Surg 11(1): 54-60.

6. Su S, Bell D, Hanna E (2014) Esthesioneuroblastoma, Neuroendocrine Carcinoma, and Sinonasal Undifferentiated Carcinoma: Differentiation in Diagnosis and Treatment. Int Arch Otorhinolaryngol 18(S02): S149156.

7. Frierson HF, Mills SE, Fechner RE, Taxy JB, Levine PA (1986) Sinonasal undifferentiated carcinoma. An aggressive neoplasm derived from schneiderian epithelium and distinct from olfactory neuroblastoma. Am J Surg Pathol 10(11): 771-779.

8. Reiersen DA, Pahilan ME, Devaiah AK (2012) Meta-analysis of treatment outcomes for sinonasal undifferentiated carcinoma. Otolaryngol-Head Neck Surg 147(1): 7-14

9. Wenig BM (2009) Undifferentiated malignant neoplasms of the sinonasal tract. Arch Pathol Lab Med 133(5): 699-712.

10. Rosenthal DI, Barker JL, El-Naggar AK, Glisson BS, Kies MS, et al. (2004) Sinonasal malignancies with neuroendocrine differentiation: patterns of failure according to histologic phenotype. Cancer 101(11): 25672573.

11. Musy PY, Reibel JF, Levine PA (2002) Sinonasal undifferentiated carcinoma: the search for a better outcome. The Laryngoscope $112(8$ Pt 1): 1450-1455.

12. Al-Mamgani A, van Rooij P, Mehilal R, Tans L, Levendag PC (2013) Combined-modality treatment improved outcome in sinonasal undifferentiated carcinoma: single-institutional experience of 21 patients and review of the literature. Eur Arch Otorhinolaryngol 270(1): 293-299.

13. Tanzler ED, Morris CG, Orlando CA, Werning JW, Mendenhall WM (2009) Management of sinonasal undifferentiated carcinoma. Head Neck 30(5): 595-599.

14. Chen AM, Daly ME, El-Sayed I, Garcia J, Lee NY, et al. (2008) Patterns of failure after combined-modality approaches incorporating radiotherapy for sinonasal undifferentiated carcinoma of the head and neck. Int J Radiat Oncol Biol Phys 70(2): 338-343.

15. Xu CC, Dziegielewski PT, McGaw WT, Seikaly H (2013) Sinonasal undifferentiated carcinoma (SNUC): the Alberta experience and literature review. J Otolaryngol Head Neck Surg 42: 2.

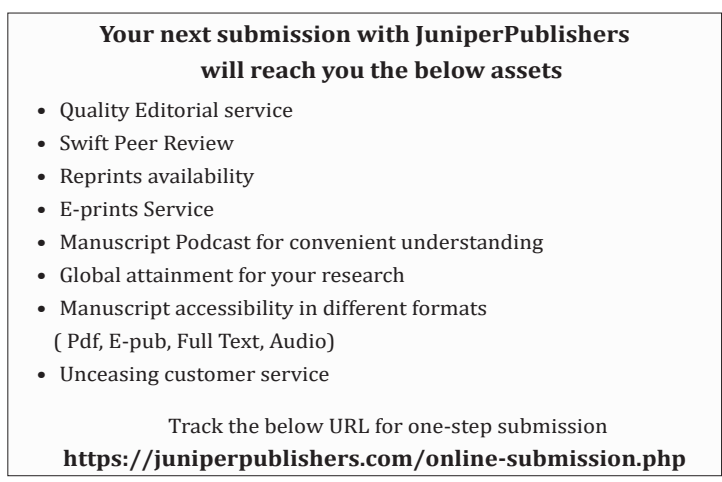

